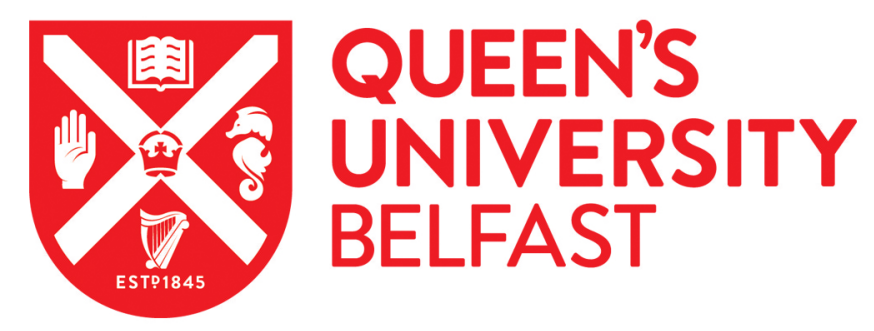

\title{
Using the Proton Energy Spectrum and Microdosimetry To Model Proton Relative Biological Effectiveness
}

Newpower, M., Patel, D., Bronk, L., Guan, F., Chaudhary, P., McMahon, S. J., Prise, K. M., Schettino, G., Grosshans, D. R., \& Mohan, R. (2019). Using the Proton Energy Spectrum and Microdosimetry To Model Proton Relative Biological Effectiveness. International journal of radiation oncology, biology, physics. https://doi.org/10.1016/j.ijrobp.2019.01.094

Published in:

International journal of radiation oncology, biology, physics

Document Version:

Peer reviewed version

Queen's University Belfast - Research Portal:

Link to publication record in Queen's University Belfast Research Portal

Publisher rights

Copyright 2019 Elsevier.

This manuscript is distributed under a Creative Commons Attribution-NonCommercial-NoDerivs License

(https://creativecommons.org/licenses/by-nc-nd/4.0/), which permits distribution and reproduction for non-commercial purposes, provided the author and source are cited.

\section{General rights}

Copyright for the publications made accessible via the Queen's University Belfast Research Portal is retained by the author(s) and / or other copyright owners and it is a condition of accessing these publications that users recognise and abide by the legal requirements associated with these rights.

Take down policy

The Research Portal is Queen's institutional repository that provides access to Queen's research output. Every effort has been made to ensure that content in the Research Portal does not infringe any person's rights, or applicable UK laws. If you discover content in the Research Portal that you believe breaches copyright or violates any law, please contact openaccess@qub.ac.uk. 


\section{Accepted Manuscript}

Using the Proton Energy Spectrum and Microdosimetry To Model Proton Relative Biological Effectiveness

Mark Newpower, MS, Darshana Patel, PhD, Lawrence Bronk, PhD, Fada Guan, PhD, Pankaj Chaudhary, PhD, Stephen J. McMahon, PhD, Kevin M. Prise, PhD, Giuseppe Schettino, PhD, David R. Grosshans, MD, PhD, Radhe Mohan, PhD

PII: S0360-3016(19)30185-3

DOI: https://doi.org/10.1016/j.jijrobp.2019.01.094

Reference: $\quad$ ROB 25524

To appear in: International Journal of Radiation Oncology $・$ Biology $\bullet$ Physics

Received Date: 30 August 2018

Revised Date: 28 December 2018

Accepted Date: 29 January 2019

Please cite this article as: Newpower M, Patel D, Bronk L, Guan F, Chaudhary P, McMahon SJ, Prise KM, Schettino G, Grosshans DR, Mohan R, Using the Proton Energy Spectrum and Microdosimetry To Model Proton Relative Biological Effectiveness, International Journal of Radiation Oncology • Biology • Physics (2019), doi: https://doi.org/10.1016/j.jirobp.2019.01.094.

This is a PDF file of an unedited manuscript that has been accepted for publication. As a service to our customers we are providing this early version of the manuscript. The manuscript will undergo copyediting, typesetting, and review of the resulting proof before it is published in its final form. Please note that during the production process errors may be discovered which could affect the content, and all legal disclaimers that apply to the journal pertain. 


\section{Using the Proton Energy Spectrum and Microdosimetry To Model Proton Relative Biological Effectiveness}

Short title: Proton MKM fitting

Mark Newpower, MS, ${ }^{* \dagger}$ Darshana Patel, PhD, ${ }^{*}$ Lawrence Bronk, PhD, ${ }^{\ddagger}$ Fada Guan, PhD,* Pankaj Chaudhary, PhD,${ }^{\S}$ Stephen J. McMahon, PhD,${ }^{\S}$ Kevin M. Prise, $\mathrm{PhD},{ }^{\S}$ Giuseppe Schettino, PhD, ${ }^{\|, ~}$ II David R. Grosshans, MD, PhD, ${ }^{\ddagger}$ and Radhe Mohan PhD, ${ }^{*}$

* Department of Radiation Physics, The University of Texas MD Anderson Cancer Center, Houston, TX, USA

${ }^{\dagger}$ Medical Physics Program, The University of Texas Graduate School of Biomedical Sciences at Houston, Houston, TX, 77030

${ }^{\ddagger}$ Department of Experimental Radiation Oncology, The University of Texas MD Anderson Cancer Center, Houston, TX, USA

${ }^{\S}$ Centre for Cancer Research and Cell Biology, School of Medicine, Dentistry and Biomedical Sciences, Queen's University Belfast, Belfast, UK

" National Physical Laboratory, Hampton Road, Teddington, Middlesex, Tw11 0LW, UK

${ }^{\mathbb{I}}$ University of Surrey, Department of Physics, 388 Stag Hill, Guilford, GU2 7XH, UK

\#Department of Radiation Oncology, The University of Texas MD Anderson Cancer Center, Houston, TX, USA

Statistical analysis performed by: Mark Newpower, M.S. Department of Radiation Physics, The 
University of Texas MD Anderson Cancer Center, Houston, TX 77030 Email:

manewpower@mdanderson.org.

Corresponding Author: Mark Newpower, M.S. Department of Radiation Physics, The University of Texas MD Anderson Cancer Center, Houston, TX 77030. Tel: (+1) 713-745-3677; Email: manewpower@mdanderson.org.

Conflict of Interest: None.

Acknowledgements: The authors thank Oleg Vassiliev for his contributions to this work and for Tamara Locke in the Scientific Publications Department at MD Anderson for editing the manuscript. This study was supported in part by the NIH/NCI grant 2U19CA021239. 


\title{
Using the Proton Energy Spectrum and Microdosimetry to Model Proton Relative Biological Effectiveness
}

\begin{abstract}
Purpose: We introduce a methodology to calculate the microdosimetric quantity dose-mean lineal energy for input into the microdosimetric kinetic model (MKM) to model the relative biological effectiveness (RBE) of proton irradiation experiments.
\end{abstract}

Methods and Materials: The data from seven individual proton RBE experiments were included in this study. In each experiment, the RBE at several points along the Bragg curve was measured. Monte Carlo (MC) simulations to calculate the lineal energy probability density function of 172 different proton energies were carried out with use of Geant4 DNA. We calculated the fluence-weighted lineal energy probability density function $\left(f_{w}(y)\right)$, based on the proton energy spectra calculated through MC at each experimental depth, calculated $\overline{y_{D}}$ for input into the MKM, and then computed the RBE. The radius of the domain $\left(r_{d}\right)$ was varied to reach the best agreement between the MKM-predicted RBE and experimental RBE. A generic RBE model as a function of dose averaged linear energy transfer $\left(\mathrm{LET}_{\mathrm{D}}\right)$ with one fitting parameter was presented and fit to the experimental RBE data as well, to facilitate a comparison to the MKM.

Results: Both the MKM and $\mathrm{LET}_{\mathrm{D}}$ based models modeled the RBE from experiments well. Values for $r_{d}$ were similar to those of other cell lines under proton irradiation that were modeled with the MKM. Analysis of the performance of each model revealed neither model was clearly superior to the other.

Conclusions: Our three key accomplishments include the following: (1) Developed a method that uses the proton energy spectra and lineal energy distributions of those protons to calculate 
dose-mean lineal energy. (2) Demonstrated that our application of the MKM provides theoretical validation of proton irradiation experiments that show that $\mathrm{RBE}$ is significantly greater than 1.1 . (3) Showed that there is no clear evidence that the MKM is better than LET $_{\mathrm{D}}$ based RBE models.

\section{Introduction}

With increased adoption of proton therapy in clinics around the world, researchers are increasingly focusing on the issue of relative biological effectiveness (RBE). In proton therapy, $\mathrm{RBE}$ is defined as the ratio of $\mathrm{x}$-ray dose to proton dose needed to reach a specified biological end point, most often clonogenic cell survival. Based on early experiments, and summarized by Paganetti et al. [1], RBE in proton therapy was set to a constant value of 1.1 at all points along the beam and for all end points. Experiments, however, have shown a wide range of RBE values and demonstrate RBE's dependency on factors such as dose, cell type, point in the cell cycle, biological end point, etc. An updated, comprehensive study of proton RBE performed by Paganetti [2], using the available experimental data from published literature, revealed that due to a lack of standardization across experiments and large uncertainties, RBE values on average are $\sim 1.1$ but increase at the end of the proton beam's range. This increased RBE at the end of the range may have clinical consequences, as demonstrated by Peeler et al. and Underwood et al. [3, 4]. Thus, it is important to take into account variable RBE during the treatment planning process. Several phenomenological RBE models have been developed [5-11], based on in vitro experiments, in order to model the observed variable RBE. Dose-averaged linear energy transfer $\left(\mathrm{LET}_{\mathrm{D}}\right)$ is often used as input for these models. However, as summarized by Mohan et al. [12], for a broad spectrum of proton energies, protons with higher LET should, in theory, contribute more to cell killing than do the lower LET protons in the spectrum for the same dose deposited if RBE is not linear with LET. At the Bragg peak and distal edge of a proton beam, the proton 
energy spectrum is indeed broad, meaning there is a correspondingly large spread in LET. This large variability in LET could make use of $\mathrm{LET}_{\mathrm{D}}$ and the reference radiation $\alpha$ and $\beta$ values alone an inappropriate measure of biological effect. One purpose of this study was to compare an RBE model as a function of $\mathrm{LET}_{\mathrm{D}}$ to that of the MKM. This allows the comparison of RBE models with different underlying expressions for radiation quality: $\mathrm{LET}_{\mathrm{D}}$ and $\overline{y_{D}}$.

The microdosimetric kinetic model (MKM) proposed by Hawkins [13, 14], in contrast, uses lineal energy probability density function $f(y)$ as an input. In this work, we present a method with which to calculate $f(y)$ for a monoenergetic proton beam with use of Monte Carlo simulation. Then, we calculated a fluence-weighted lineal energy probability density function $\left(f_{w}(y)\right)$ for a polyenergetic proton beam, calculated the dose mean lineal energy $\left(\overline{y_{D}}\right)$ and used this as input into the MKM to predict RBE. Finally, we varied a fitting parameter in the MKM to achieve a best-fit between the MKM and RBE experiments. Our work showed that the MKM can be applied to explain the increase in RBE as a function of $\overline{y_{D}}$ in recent proton irradiation experiments. To compare the MKM fitting of RBE as a function of $\overline{y_{D}}$ to an RBE model that is a function of $\mathrm{LET}_{\mathrm{D}}$, we propose a generic RBE model with one fitting parameter. This parameter was varied to achieve the best fit to experimental data, and then the generic RBE model and the MKM fits were compared using Akaike Information Criterion (AIC) [15] to determine which of the two models best described the data.

\section{Methods and Materials}

\section{RBE data}

The RBE data and proton energy spectra corresponding to the RBE measurements were obtained from the three studies by Chaudhary and Marshall et al. [16], Guan and Bronk et al. [17], and Patel and Bronk et al. [18]. In all three studies, the authors measured RBE at various depths 
along a proton beam. In addition, they simulated their beam geometry with Geant4 [19], calculating $\mathrm{LET}_{\mathrm{D}}$ and the proton energy spectrum at each measurement point. Together, these three studies represent seven total experiments across three different beamlines and four cell lines. Pristine Bragg peaks were used for all three studies, and Chaudhary and Marshall et al. also included spread-out Bragg peak investigations in their study.

\section{Lineal energy $(y)$ :}

Lineal energy $y$ is defined as the quotient of the energy deposited into a volume of interest $\varepsilon$ and the mean chord length through that volume $\bar{l}$ by a single energy deposition event [20], and is expressed as

$$
y=\frac{\varepsilon}{\bar{l}}
$$

In this work the volume of interest is called the domain and is defined as a sphere with radius $r_{d}$. In microdosimetry, $y$ is a stochastic quantity that follows a distribution, called the lineal energy probability density function $(f(y))$ [21]. The second moment of $f(y)$ is the dose-mean lineal energy and is expressed as

$$
\overline{y_{D}}=\frac{\int y^{2} f(y) d y}{\int y f(y) d y} .
$$

\section{Calculating $y$}

Geant4 DNA version 10.2.0 was used to calculate the $f(y)$ for 172 proton energies. The basis for the selection was to ensure adequate sampling of $y$ values at all proton energies used in the RBE experiments. The Geant4 DNA extension $[22,23]$ was used to simulate 100 tracks at each energy in a liquid water cube geometry of 5 - $\mu \mathrm{m}$ side lengths. $5-\mu \mathrm{m}$ was chosen to match the voxel size that $\mathrm{LET}_{\mathrm{D}}$ was scored in. To calculate $f(y)$ at a particular energy, a Matlab script was 
developed to score the lineal energy $y$ at randomly generated locations around the proton track, inside spherical domains with a radius $\left(r_{d}\right)$ of $500 \mathrm{~nm}$ for each track. The scored $y$ values were then binned into a histogram with a bin width of $0.1 \mathrm{keV} / \mu \mathrm{m}$, with a range of $[0.01,300]$ $\mathrm{keV} / \mu \mathrm{m}$.

\section{Calculating $\overline{y_{D}}$}

For a proton of a given energy, the lineal energy follows a distribution rather than holding a single, fixed value. Therefore, to get an accurate account of the energy loss across a spectrum of proton energies, the lineal energy probability density function of a polyenergetic proton beam $\left(f_{w}(y)\right)$ has to take into account the various constituent $f(y)$ values and their respective weights in the proton energy spectrum. We calculated $f_{w}(y)$ with use of a fluence-weighting technique. At each point along the Bragg curve where RBE was measured, the proton energy fluence $(\Phi(E))$ was calculated via Monte Carlo simulation. Using the fluence at energy $i$ and $f(y)_{i}, f_{w}(y)$ was calculated as follows:

$$
f_{w}(y)=\frac{\sum_{i} \Phi(E)_{i} f(y)_{i}}{\sum_{i} \Phi(E)_{i}}
$$

The calculation of $\overline{y_{D}}$ for a polyenergetic beam is then straightforward

$$
\overline{y_{D}}=\frac{\int y^{2} f_{w}(y) d y}{\int y f_{w}(y) d y} .
$$

\section{Uncertainty analysis of $\boldsymbol{f}(\boldsymbol{y})$}

We assumed that the number of scored $y$ values in each bin follows a Poissonian distribution,

and the fractional error per bin can be calculated as $\frac{\sqrt{n \text { samples }}}{n \text { samples }}$. Since calculating $\overline{y_{D}}$ is essentially a sum over all scored $y$ values, as shown in Eqs. 2 and 4, the total fractional error of each $\overline{y_{D}}$ value is the quadrature sum of the fractional errors from each bin, divided by the sum of 
all $y$ samples scored. The number of scores per track was increased until the maximum fractional uncertainty for any $f(y)$ was approximately $1.5 \times 10^{-4}$. Table $\mathrm{S} 2$ contains the number of scores per track that was performed.

\section{Modeling RBE with the MKM}

MKM builds upon the theory of dual radiation action, which was proposed by Kellerer and Rossi [24]. It uses the linear-quadratic (LQ) framework and predicts the $\alpha$ parameter as a function of $\overline{y_{D}}$. We calculated $\alpha_{p}$ by using the method proposed by Kase et al. [25]:

$$
\alpha_{p}=\alpha_{x}+\beta_{x} \frac{\overline{y_{D}}}{\rho \pi r_{d}^{2}}
$$

where $\alpha_{x}$ and $\beta_{x}$ are the linear and quadratic fitting parameters from the reference radiation survival curve, $\rho$ is the mass density of water and $r_{d}$ is the radius of the domain. Eqs. 3 and 4 show how we calculated $\overline{y_{D}}$ at each point along the Bragg curve in each experiment. Since RBE is defined as the ratio of doses of two radiation types to reach a specified biological endpoint - in this case, a given surviving fraction (SF) - we calculate RBE by solving for both doses from the LQ model. To calculate the experimental RBE, we use the LQ parameters from each clonogenic survival experiment from Refs. 16-18. The values used for $\alpha_{x}$ and $\beta_{x}$ are given in S1 and the reader is referred to Refs. $16-18$ for the LQ parameters for the proton experiments. The MKM assumes $\beta_{p}=\beta_{x}$, and $\alpha_{p}$ is calculated from Eq. 5. Thus RBE can be calculated by solving the LQ model $S F=e^{-\alpha D-\beta D^{2}}$ for the corresponding dose in each irradiation modality, giving

$$
R B E=\frac{\sqrt{\alpha_{x}^{2}-4 \beta_{x} \ln S F}-\alpha_{x}}{\sqrt{\alpha_{p}^{2}-4 \beta_{p} \ln S F-\alpha_{p}}} \times \frac{\beta_{p}}{\beta_{x}} .
$$

Other works have derived similar expressions for RBE as a function of dose or survival fraction 
[7]. In this work, all RBE values were calculated for a survival fraction (SF) of $10 \%$. To find the best $r_{d}$ for each experiment, $r_{d}$ was varied in Eq. 5 through the least squares method. We assume that $\overline{y_{D}}$ is independent of $r_{d}$ for sufficiently small domains so that even changing the value of $r_{d}$ on the order of a 200-300 nanometers will not change the underlying microdosimetric distributions.

\section{Comparing the MKM to a generic RBE model}

In order to compare the MKM with a model based on $\mathrm{LET}_{\mathrm{D}}$ we propose an RBE model whose $\beta$ is constant $\left(\beta_{p}=\beta_{x}\right)$ and whose $\alpha$ parameter varies linearly with LET $_{\mathrm{D}}$ :

$$
\alpha_{p}=\alpha_{x}+k \times L E T_{D}
$$

This approach is similar to the one taken by Chaudhary and Marshall et al. [16]. This model and the MKM are only approximately correct because the $\mathrm{LET}_{\mathrm{D}}$ of an $\mathrm{x}$-ray beam is nonzero, around $0.2 \mathrm{keV} / \mu \mathrm{m}$. However as LET $_{\mathrm{D}}$ increases, Eq. 5 and Eq. 7 become a better model of biological response. We applied this model to each RBE experiment and varied the $k$ term to reach a best fit for the data, through the least squares method. The sum of squares from each model and experiment combination were input into the Akaike Information Criterion [15] to determine which model best describes the data.

\section{Results}

\section{Calculating $\overline{y_{D}}$}

The proton energy spectrum at each point along the Bragg curve for each experiment was used to calculate both the $\operatorname{LET}_{\mathrm{D}}$ and $\overline{y_{D}}$, with $\overline{y_{D}}$ being calculated according to Eq. 3 and 4 , and the resulting relationship between the two quantities is noted in Fig. 2. For each experiment, $\overline{y_{D}}$ appears to be an approximately linear function of $\mathrm{LET}_{\mathrm{D}}$. 


\section{Uncertainty analysis of $f(y)$}

In Fig. 1a, the fractional errors for each $f(y)$ scored is plotted. We input each of our scored $f(y)$ functions into Eq. 2, calculated $\overline{y_{D}}$, and compared our $\overline{y_{D}}$ to those reported by Nikjoo et al. [26] in Fig. 1b.

\section{Modeling RBE with MKM}

By varying $r_{d}$, we were able to fit the experimental RBE data well by using the MKM, as shown in Figs. 3-5. Values for the best-fit $r_{d}$ are shown on the plots and in Table 1. Note that in Figs. 35, the RBE modeled by the MKM is plotted as a function of LET $_{\mathrm{D}}$, even though the MKM is a function of $\overline{y_{D}}$. This was done to be consistent with past practice and to enable plotting both the MKM and generic RBE model on one plot. While Fig. 2 shows the linear fit for $\overline{y_{D}}$ as a function of $\operatorname{LET}_{\mathrm{D}}$ for each beamline, $\overline{y_{D}}$ values were not directly transformed via these equations. Rather, the points were plotted directly against the calculated LET $_{D}$ values.

\section{Comparing the MKM to a generic RBE model}

Table 1 contains the values of each of the $k$ parameters for the generic RBE model given in Eq.

7. The output of the AIC calculation is the relative likelihood that a given model is a better fit than another model and are given in Table 1. In two experiments, the MKM was found to fit the data better than the generic RBE model, while five experiments were better modeled by the generic RBE model. However, in the majority of the experiments the AIC analysis also suggests that there is little evidence to choose between the models as the differences in AICs were typically small.

\section{Discussion}

In the present study we calculated $\overline{y_{D}}$ at various depths along the Bragg curve, based on the proton energy spectra at that depth. By inputting $\overline{y_{D}}$ at various depths along the Bragg curve into 
the MKM and varying $r_{d}$ we were able to find a best-fit $r_{d}$ that modeled the experimental RBE data well. We then compared this fit of the MKM to a generic RBE model with one fitting parameter to determine if RBE was better modeled by the MKM or a generic RBE model that includes $\mathrm{LET}_{\mathrm{D}}$ as a measure of radiation quality. AIC analysis indicated that for two experiments, MKM was the superior model and for five experiments, the generic RBE model was better. However in most experiments there was not strong evidence favoring either model.

The values published by Nikjoo et al. [26] provide a useful reference for our $\overline{y_{D}}$ values, since both our method and Nikjoo et al.'s published data are modeled with use of a domain radius of $500 \mathrm{~nm}$. When comparing our values to those of Nikjoo et al. in Fig. 1b), we see excellent agreement except the lowest two proton energies, 0.1 and $0.2 \mathrm{MeV}$. However these energies contribute so little dose in a clinical proton beam spectrum that they do not have a significant impact on our microdosimetric calculations. Our $\overline{y_{D}}$ calculations also agree well with those reported by Lindborg et al [27]. The low fractional error and agreement with the values published by Nikjoo et al. and Lindborg et al. give us confidence in the accuracy of the values of our calculations for $\overline{y_{D}}$. Figure 2 shows that $\overline{y_{D}}$ and $\operatorname{LET}_{\mathrm{D}}$ increase in a linear relationship. This relationship has been derived by Kellerer and is $\overline{y_{D}}=\frac{9}{8} L E T_{D}+\frac{3 \delta_{2}}{2 d}$ where $\delta_{2}$ is the weighted energy loss per collision and $d$ is the diameter of the domain [28].Confidence intervals for the linear relationships between $\overline{y_{D}}$ and $\mathrm{LET}_{\mathrm{D}}$ plotted in Fig. 2 are reported in the Supplementary Data. Recently, a series of microdosimetric measurements were conducted by Anderson et al. [29] where they measured $\overline{y_{D}}$ at various depths along a 71.3 MeV and $159.9 \mathrm{MeV}$ proton beam and compared those measurements to calculations of $\mathrm{LET}_{\mathrm{D}}$. They found a similar, linear relationship between $\mathrm{LET}_{\mathrm{D}}$ and $\overline{y_{D}}$, and our values of $\overline{y_{D}}$ at similar $\operatorname{LET}_{\mathrm{D}}$ are similar to theirs. It is important to highlight the differences between $\mathrm{LET}_{\mathrm{D}}$ and $\overline{y_{D}}$. $\mathrm{LET}_{\mathrm{D}}$ is calculated by sampling 
the stopping power, known as the unrestricted LET, of each proton that passes through the scoring volume and averaging the LET values by weighing them according to how much energy was deposited by those protons in the scoring volume. $\overline{y_{D}}$ is a measure of the energy deposited into a volume, called a domain. The energy deposited into the domain can come from the primary proton and delta rays. When calculating $\overline{y_{D}}$, we include instances when the proton passes near the domain, but not through it, and delta rays deposit energy into the domain.

In this study, $r_{d}$ was varied for each experiment to achieve a best fit between the MKM and experiments. The values for $r_{d}$ for these experiments range between $0.202 \mu \mathrm{m}$ and 0.461 $\mu \mathrm{m}$. Work by Kase et al. [30] showed that when fitting the MKM to other proton RBE data, $r_{d}$ values were $0.26 \mu \mathrm{m}, 0.34 \mu \mathrm{m}$, and $0.35 \mu \mathrm{m}$ for V79, HSG, and T1 cell lines, respectively. Mairani et al. [31] conducted a similar fitting utilizing proton and helium ion irradiation data and found their best fitting $r_{d}$ value was $0.300 \mu \mathrm{m}$. We concluded that our $r_{d}$ values were similar to previous studies that applied the MKM to proton RBE experiments. Patel and Bronk and Guan and Bronk used the same cell line, H460, in their experiments. We were able to fit both of them by using the MKM. However, the value of $r_{d}$ to get a best fit was different for each experiment. Based on Guan et al.'s data, $r_{d}$ was $0.323 \mu \mathrm{m}$ and for Patel et al.'s data, $r_{d}$ was $0.426 \mu \mathrm{m}$. The Guan and Bronk et al. data show RBE as a much more nonlinear function of $\mathrm{LET}_{\mathrm{D}}$ than the similar studies performed by Patel and Bronk et al. and Chaudhary and Marshall et al., and it remains unclear why. Both the generic RBE model and the MKM fit this data set poorly when compared to the fits shown in Figs. 4 and 5. Three RBE models were applied to the Guan and Bronk et al. data by McNamara et al. [9], and Mohan et al. [12] and their models were unable to account for the highest experimental RBE, though their models were better able to fit the RBE at $15 \mathrm{keV} / \mu \mathrm{m}$ and below. The study in Ref. 18 highlights the importance and sensitivity of the 
settings used in Monte Carlo simulations, particularly when the $\mathrm{LET}_{\mathrm{D}}$ and dose values from simulations are used for calculating RBE. We suggest that the difference in Monte Carlo settings in the different studies is the reason for the large difference in $r_{d}$ values for the same cell line in two different experiments, and why both the MKM and generic RBE model fit the Patel and Bronk et al. data, and Chaudhary and Marshall et al. data better. In the high gradient region distal to the Bragg peak, there may be significant uncertainties in delivered dose, which may be quite different among the different experiments. To address these issues, we are further investigating the relationship between $\mathrm{RBE}, \overline{y_{D}}$ and $\mathrm{LET}_{\mathrm{D}}$ by performing cell irradiation experiments using ramp fields and SOBPs with different dose and $\operatorname{LET}_{D}$ combinations $[32,33]$. What is also striking about the experimental data in Figs. 3 and 4 is the RBE at $\mathrm{LET}_{\mathrm{D}}>\sim 15 \mathrm{keV} / \mu \mathrm{m}$ seems to be increasing nonlinearly. This is in contrast to heavy ion studies that show RBE as a linear function of $\mathrm{LET}_{\mathrm{D}}$ up to around $100 \mathrm{keV} / \mu \mathrm{m}$ [34]. This nonlinearity at high proton $\mathrm{LET}_{\mathrm{D}}$ may also point to modifications of the MKM and other RBE models, such as including a variable $\beta$ term or higher-order terms in the relationship between LET and $\alpha$.

The results of fitting the MKM and the generic RBE model to the experimental data are shown in Figs. 3-5. Overall, both models gave very similar fits to the data. Particularly in the U87 and AGO1522 pristine Bragg peak experiments in the Chaudhary and Marshall et al. data, and the Patel and Bronk et al. data, RBE appears to be a linear function of LET $_{D}$. For the SOBP experiments from Chaudhary and Marshall et al., the MKM better models the RBE at the lowest three $\mathrm{LET}_{\mathrm{D}}$ points. The difference in the two models can be significant, as seen in Fig. 5b) and $5 d)$. Others have suggested that $\operatorname{LET}_{\mathrm{D}}$ may not be the best surrogate for biological response [12]. The superior fit of the MKM shown in Fig. 5b) and 5d) support the hypothesis that $\overline{y_{D}}$ better encapsulates biological effect than LET $_{\mathrm{D}}$, particularly in an SOBP, supporting the soundness of 
its underlying assumptions. However, more studies similar to the one presented here are needed to fully determine the relative merits of MKM or $\mathrm{LET}_{\mathrm{D}}$ based models in a proton SOBP. To determine which RBE model fits the data best, the AIC analysis in Table 1 was used. Overall, the generic RBE model was preferred in five experiments and the MKM in two. A minor caveat is the relatively weak preference for the generic RBE model in the Guan and Bronk et al. data, where the relative likelihood of MKM being the better model was 0.140 and 0.163 for the $\mathrm{H} 460$ and H1437 experiments, respectively.

Based on the AIC analysis between the generic RBE model and the MKM, there is no clear evidence that the MKM models RBE better than a generic $\mathrm{LET}_{\mathrm{D}}$ based model. We have demonstrated that the MKM can be used to model proton RBE experiments whose RBE values are much higher than the clinically accepted $\mathrm{RBE}=1.1$ model, however we have not demonstrated a clear superiority by using the MKM. Thus we conclude that there is not enough evidence to move away from $\mathrm{LET}_{\mathrm{D}}$ based RBE models. There may be some end of range proton RBE nonlinearity but at present we conclude that both the MKM and LET $_{D}$ based models provide an accurate model of RBE, even if the underlying RBE model is more complex.

An advantage of this MKM fitting procedure is that its use is not restricted to protons. Any radiation type, such as helium or carbon, whose $f_{w}(y)$ can be calculated, can apply this method to modeling RBE. A modified version of the MKM was incorporated into a treatment planning system for carbon ions by Inaniwa et al. [35] and our group is developing this MKM algorithm to be implemented in a treatment planning system for proton therapy. Future studies will examine the differences in treatment plans optimized by using the traditional $\mathrm{RBE}=1.1$ paradigm and those optimizing on biological effect by using the MKM.

\section{Conclusions}


Using data from three independent proton RBE studies, we have presented a method to calculate the dose mean lineal energy $\left(\overline{y_{D}}\right)$ of a polyenergetic proton beam and apply the microdosimetric kinetic model (MKM) to the RBE experiments. To our knowledge, this fluence-based method of calculating the probability density function $f_{w}(y)$ based on the proton energy distribution and $f(y)$ for monoenergetic proton beams is unique. This study provides a theoretical basis for modeling the increase in RBE along the distal edge of a proton beam. We also applied a generic $\mathrm{LET}_{\mathrm{D}}$ based RBE model to the same data and compared the MKM and generic RBE model fittings with AIC analysis. Based on this analysis there is no clear evidence that the MKM, using radiation quality represented by $\overline{y_{D}}$, is superior to a generic model using $\operatorname{LET}_{\mathrm{D}}$ as a measure of the radiation quality. Given the wide ranges of LET values in a spread out Bragg peak and the concern about the quantity $\operatorname{LET}_{\mathrm{D}}$, measuring $f(y)$ distributions and $\overline{y_{D}}$ with a tissue equivalent proportional counter may be the preferred way to characterize proton beam quality in the clinic, at least as a calibration of Monte Carlo simulation settings.

\section{References}

1. Paganetti, H., A. Niemierko, M. Ancukiewicz, et al., Relative biological effectiveness $(R B E)$ values for proton beam therapy. Int J Radiat Oncol Biol Phys, 2002. 53(2): p. 407421.

2. Paganetti, H., Relative biological effectiveness ( $R B E$ ) values for proton beam therapy. Variations as a function of biological endpoint, dose, and linear energy transfer. Phys Med Biol., 2014. 419(59): p. R419-R472.

3. Peeler, C.R., D. Mirkovic, U. Titt, et al., Clinical evidence of variable proton biological effectiveness in pediatric patients treated for ependymoma. Radiother Oncol, 2016. 121(3): p. 395-401.

4. Underwood, T., C. Grassberger, R. Bass, et al., OC-0245: Clinical evidence that end-ofrange proton $R B E$ exceeds 1.1: lung density changes following chest $R T$. Radiother Oncol, 2017. 123: p. S123-S124.

5. Abolfath, R., C.R. Peeler, M. Newpower, et al., A model for relative biological effectiveness of therapeutic proton beams based on a global fit of cell survival data. Sci Rep, 2017. 7(1): p. 8340. 
6. Carabe-Fernandez, A., R.G. Dale, and B. Jones, The incorporation of the concept of minimum RBE (RBEmin) into the linear-quadratic model and the potential for improved radiobiological analysis of high-LET treatments. Int J of Radiat Biol, 2007. 83(1): p. 2739.

7. Chen, Y. and S. Ahmad, Empirical model estimation of relative biological effectiveness for proton beam therapy. Radiat Prot Dosimetry, 2012. 149(2): p. 116-23.

8. Jones, B., Towards Achieving the Full Clinical Potential of Proton Therapy by Inclusion of LET and RBE Models. Cancers, 2015. 7(1): p. 460-80.

9. McNamara, A.L., J. Schuemann, and H. Paganetti, A phenomenological relative biological effectiveness $(R B E)$ model for proton therapy based on all published in vitro cell survival data. Phys Med Biol, 2015. 60(21): p. 8399-416.

10. Rorvik, E., S. Thornqvist, C.H. Stokkevag, et al., A phenomenological biological dose model for proton therapy based on linear energy transfer spectra. Med Phys, 2017. 44(6): p. 2586-2594.

11. Wedenberg, M., B.K. Lind, and B. Hardemark, A model for the relative biological effectiveness of protons: the tissue specific parameter alpha/beta of photons is a predictor for the sensitivity to LET changes. Acta Oncol, 2013. 52(3): p. 580-8.

12. Mohan, R., C.R. Peeler, F. Guan, et al., Radiobiological issues in proton therapy. Acta Oncol., 2017: p. 1-7.

13. Hawkins, R.B., A microdosimetric-kinetic theory of the dependence of the RBE for cell death on LET. Med Phys, 1998. 25(7 Pt 1): p. 1157-70.

14. Hawkins, R.B., A Microdosimetric-Kinetic Model for the Effect of Non-Poisson Distribution of Lethal Lesions on the Variation of RBE with LET. Journal of radiation research, 2003. 160(1): p. 61-69.

15. Akaike, H., A new look at the statistical model identification. IEEE Trans Automat Contr, 1974. 19(6): p. 716-723.

16. Chaudhary, P., T.I. Marshall, F.M. Perozziello, et al., Relative biological effectiveness variation along monoenergetic and modulated Bragg peaks of a 62-MeV therapeutic proton beam: a preclinical assessment. Int J Radiat Oncol Biol Phys, 2014. 90(1): p. $27-$ 35.

17. Guan, F., L. Bronk, U. Titt, et al., Spatial mapping of the biologic effectiveness of scanned particle beams: towards biologically optimized particle therapy. Sci Rep, 2015. 5: p. 9850.

18. Patel, D., L. Bronk, F. Guan, et al., Optimization of Monte Carlo particle transport parameters and validation of a novel high throughput experimental setup to measure the biological effects of particle beams. Med Phys., 2017. 44(11): p. 6061-6073.

19. Agostinelli, S., J. Allison, K. Amako, et al., Geant4-a simulation toolkit. Nucl Instrum Methods Phys Res A., 2003. 506(3): p. 250-303.

20. ICRU, ICRU Report 51: Quantities and Units in Radiation Protection Dosimetry. 1993.

21. ICRU, ICRU Report 36: Microdosimetry. 1983.

22. Bernal, M.A., M.C. Bordage, J.M.C. Brown, et al., Track structure modeling in liquid water: A review of the Geant4-DNA very low energy extension of the Geant4 Monte Carlo simulation toolkit. Physica Medica, 2015. 31(8): p. 861-874.

23. Incerti, S., A. Ivanchenko, M. Karamitros, et al., Comparison of GEANT4 very low energy cross section models with experimental data in water. Med Phys., 2010. 37(9): p. 4692-4708. 
24. Kellerer, A.M. and H.H. Rossi, The theory of dual radiation action. Curr. Top. Radiat. Res. Q., 1972. 8: p. 85-158.

25. Kase, Y., T. Kanai, Y. Matsumoto, et al., Microdosimetric measurements and estimation of human cell survival for heavy-ion beams. Radiat Res, 2006. 166(4): p. 629-38.

26. Nikjoo, H., D. Emfietzoglou, T. Liamsuwan, et al., Radiation track, DNA damage and response-a review. Rep Prog Phys, 2016. 79(11): p. 116601.

27. Lindborg, L., M. Hultqvist, T. A Carlsson, et al., Lineal energy and radiation quality in radiation therapy: model calculations and comparison with experiment. Physics in Medicine \& Biology, 2013. 58(10): p. 3089.

28. Kellerer, A.M. Fundamentals of Microdosimetry. The Dosimetry of Ionizing Radiation, ed. K.R. Kase, B.E. Bjarngard, and F.H. Attix. Vol. 1. 1985, Academic Press: New York.

29. Anderson, S.E., K.M. Furutani, L.T. Tran, et al., Microdosimetric measurements of a clinical proton beam with micrometer-sized solid-state detector. Med Phys., 2017. 44(11): p. 6029-6037.

30. Kase, Y., T. Kanai, N. Matsufuji, et al., Biophysical calculation of cell survival probabilities using amorphous track structure models for heavy-ion irradiation. Phys Med Biol, 2008. 53(1): p. 37-59.

31. Mairani, A., G. Magro, T. Tessonnier, et al., Optimizing the modified microdosimetric kinetic model input parameters for proton and (4)He ion beam therapy application. Phys Med Biol, 2017. 62(11): p. N244-N256.

32. $\mathrm{XXXX}$

33. XXXX

34. Sørensen, B.S., J. Overgaard, and N. Bassler, In vitro RBE-LET dependence for multiple particle types. Acta Oncol., 2011. 50(6): p. 757-762.

35. Inaniwa, T., T. Furukawa, Y. Kase, et al., Treatment planning for a scanned carbon beam with a modified microdosimetric kinetic model. Phys Med Biol, 2010. 55(22): p. 6721-37.

\section{Figure Captions}

Fig. 1. (a) Total fractional error from each $f(y)$ scored using Geant4 DNA. (b) Comparison of our values of $\overline{y_{D}}$, using our scored probability density functions $(f(y))$, to those from Nikjoo et al [26]. (c, d) Histograms for the distribution of y values for $1 \mathrm{MeV}$ and $30 \mathrm{MeV}$ protons, respectively. These histograms were normalized and thus became the $f(y)$ functions.

Fig. 2 Plots of $\overline{y_{D}}$ as a function of $\operatorname{LET}_{\mathrm{D}}$ for each of the three experiments. Our results indicate an approximately linear relationship between $\overline{y_{D}}$ and $\mathrm{LET}_{\mathrm{D}}$. The equation of $\overline{y_{D}}$ as a linear function of $\mathrm{LET}_{\mathrm{D}}$ for each of the four beam configurations are shown in the panels. 
Fig. 3. Results of fitting the generic RBE model and the MKM, with fitting parameters. Panel a) contains the experimental data and fits for the H460 data from Guan and Bronk et al, while panel b) contains the data and fits for the H1437 data. The experimental data was performed at MDACC.

Fig. 4. Results of fitting the generic RBE model and the MKM to the Patel and Bronk et al. data. Experimental data was performed at HIT, Germany.

Fig. 5. The results of fitting the generic RBE model and the MKM for the data from Chaudhary and Marshall et al. Panels a) and c) show the pristine Bragg peak irradiations for cell lines U87 and AGO1522, respectively. Panels b) and d) show the spread out Bragg peak irradiations experiments for the U87 and AGO1522 cell lines, respectively. In the low $\mathrm{LET}_{\mathrm{D}}$ and low $\overline{y_{D}}$ region, MKM was better able to model RBE than the generic RBE model. Experiments performed at CATANA, Italy. 
Table 1. Results of AIC analysis between the MKM and generic RBE model. $r_{d}$ : radius of the domain; CI: confidence interval; SS: sum of squares; RL: relative likelihood.

\section{Guan and Bronk et al}

$\underline{\mathrm{H} 460}$

\begin{tabular}{|l|l|}
\hline \multicolumn{1}{|c|}{ MKM } & \multicolumn{1}{c|}{ Generic } \\
\hline$r_{d}=323 \mathrm{~nm}$ & $\mathrm{k}=0.0621$ \\
\hline $\mathrm{CI}=[286.7-379.3]$ & $\mathrm{CI}=[0.04798-0.07578]$ \\
\hline $\mathrm{SS} 1=1.473$ & $\mathrm{SS} 2=1.061$ \\
\hline $\mathrm{RL}=0.140$ & \multicolumn{1}{|c|}{} \\
\cline { 1 - 1 } &
\end{tabular}

H1437

\begin{tabular}{|l|l|}
\hline \multicolumn{1}{|c|}{ MKM } & \multicolumn{1}{c|}{ Generic } \\
\hline$r_{d}=310 \mathrm{~nm}$ & $\mathrm{k}=0.0330$ \\
\hline $\mathrm{CI}=[274.8-368.8]$ & $\mathrm{CI}=[0.02511-0.04073]$ \\
\hline $\mathrm{SS} 1=1.271$ & $\mathrm{SS} 2=0.9396$ \\
\hline $\mathrm{RL}=0.1632$ & \multicolumn{1}{|c}{} \\
\cline { 1 - 2 } &
\end{tabular}

\section{Patel and Bronk et al}

$\underline{\mathrm{H} 460}$

\begin{tabular}{|l|l|}
\hline \multicolumn{1}{|c|}{ MKM } & \multicolumn{1}{c|}{ Generic } \\
\hline$r_{d}=426 \mathrm{~nm}$ & $\mathrm{k}=0.045$ \\
\hline $\mathrm{CI}=[397.5-461.9]$ & $\mathrm{CI}=[0.03987-0.05014]$ \\
\hline $\mathrm{SS} 1=0.3606$ & $\mathrm{SS} 2=0.2169$ \\
\hline $\mathrm{RL}=0.0474$ & \multicolumn{2}{|c}{} \\
\cline { 1 - 1 } &
\end{tabular}

\section{Chaudhary and Marshall et al}

Pristine Bragg Peak, AGO1522

\begin{tabular}{|l|l|}
\hline \multicolumn{1}{|c|}{ MKM } & \multicolumn{1}{c|}{ Generic } \\
\hline$r_{d}=202 \mathrm{~nm}$ & $\mathrm{k}=0.08423$ \\
\hline $\mathrm{CI}=[192.4-213.9]$ & $\mathrm{CI}=[0.07092-0.09746]$ \\
\hline $\mathrm{SS} 2=0.1096$ & $\mathrm{SS} 1=0.2404$ \\
\cline { 2 - 2 } & $\mathrm{RL}=0.0948$ \\
\cline { 2 - 2 }
\end{tabular}

Pristine Bragg Peak, U87

\begin{tabular}{|l|l|}
\hline \multicolumn{1}{|c|}{ MKM } & \multicolumn{1}{c|}{ Generic } \\
\hline$r_{d}=345 \mathrm{~nm}$ & $\mathrm{k}=0.0285$ \\
\hline $\mathrm{CI}=[311.3-394.7]$ & $\mathrm{CI}=[0.02554-0.0313]$ \\
\hline
\end{tabular}




\begin{tabular}{|l|l|}
\hline $\mathrm{SS} 1=0.1189$ & $\mathrm{SS} 2=0.02386$ \\
\cline { 1 - 1 } $\mathrm{RL}=0.0081$ & \\
\cline { 1 - 2 }
\end{tabular}

$\underline{\text { SOBP, AGO1522 }}$

\begin{tabular}{|l|l|}
\hline \multicolumn{1}{|c|}{ MKM } & \multicolumn{1}{c|}{ Generic } \\
\hline$r_{d}=243 \mathrm{~nm}$ & $\mathrm{k}=0.0537$ \\
\hline $\mathrm{CI}=[227.2-262.8]$ & $\mathrm{CI}=[0.03957-0.06765]$ \\
\hline $\mathrm{SS} 2=0.1086$ & $\mathrm{SS} 1=0.3402$ \\
\cline { 2 - 2 } & $\mathrm{RL}=0.0325$ \\
\hline
\end{tabular}

$\underline{\text { SOBP, U87 }}$

\begin{tabular}{|l|l|}
\hline \multicolumn{1}{|c|}{ MKM } & \multicolumn{1}{c|}{ Generic } \\
\hline$r_{d}=461 \mathrm{~nm}$ & $\mathrm{k}=0.0147$ \\
\hline $\mathrm{CI}=[420.0-518.0]$ & $\mathrm{CI}=[0.01318-0.0194]$ \\
\hline $\mathrm{SS} 2=0.02759$ & $\mathrm{SS} 1=0.01614$ \\
\hline $\mathrm{RL}=0.2002$ & \multicolumn{1}{|c|}{} \\
\cline { 2 - 2 } &
\end{tabular}



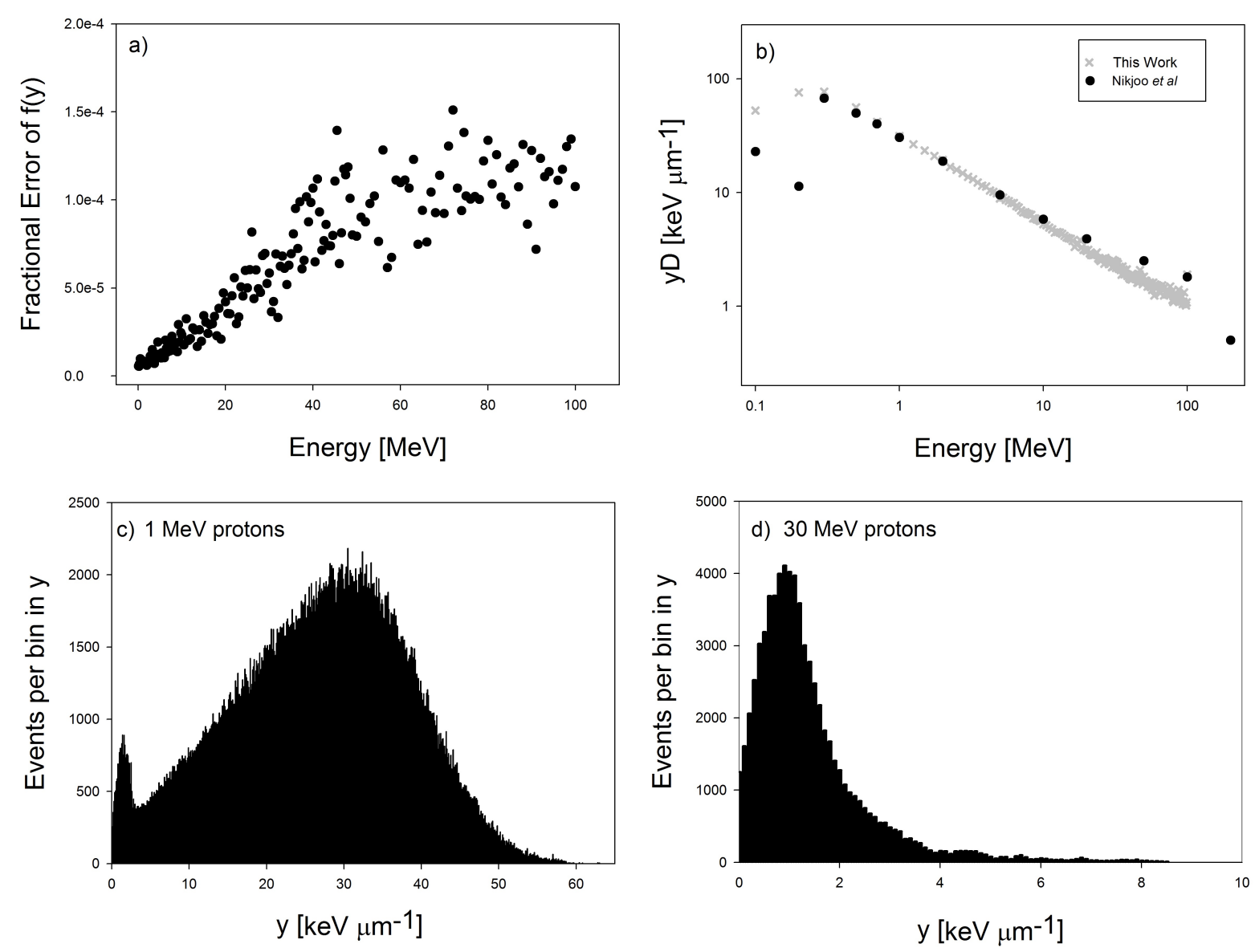

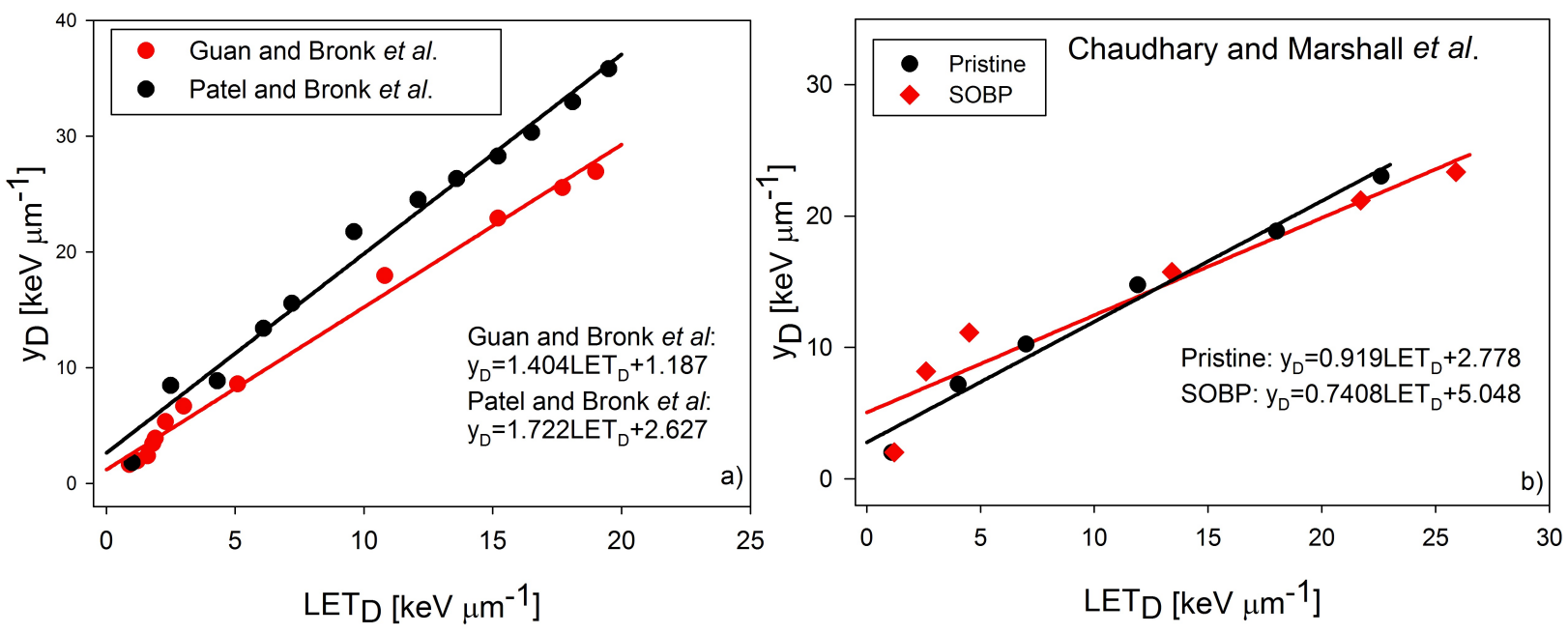

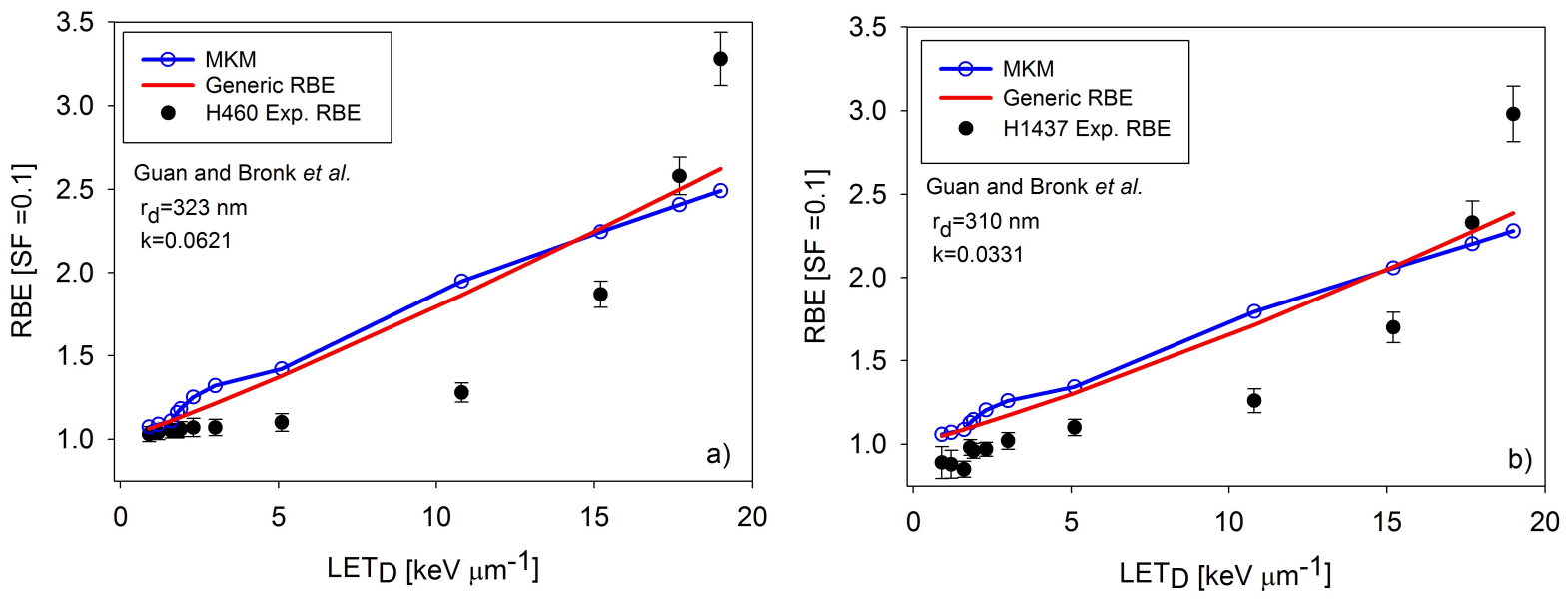


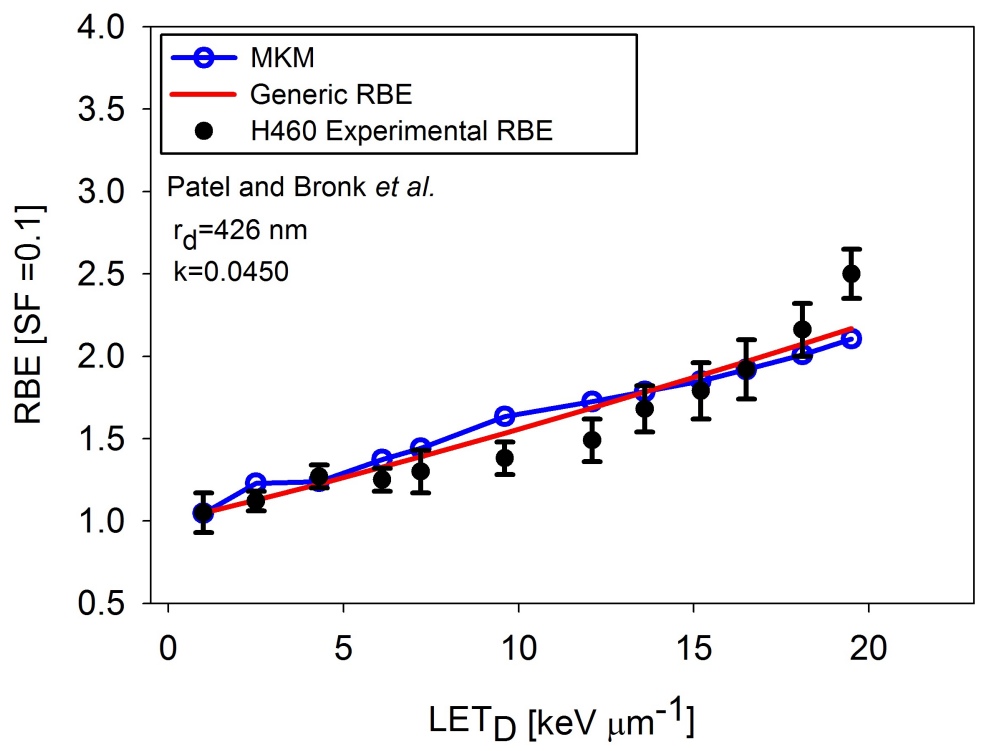



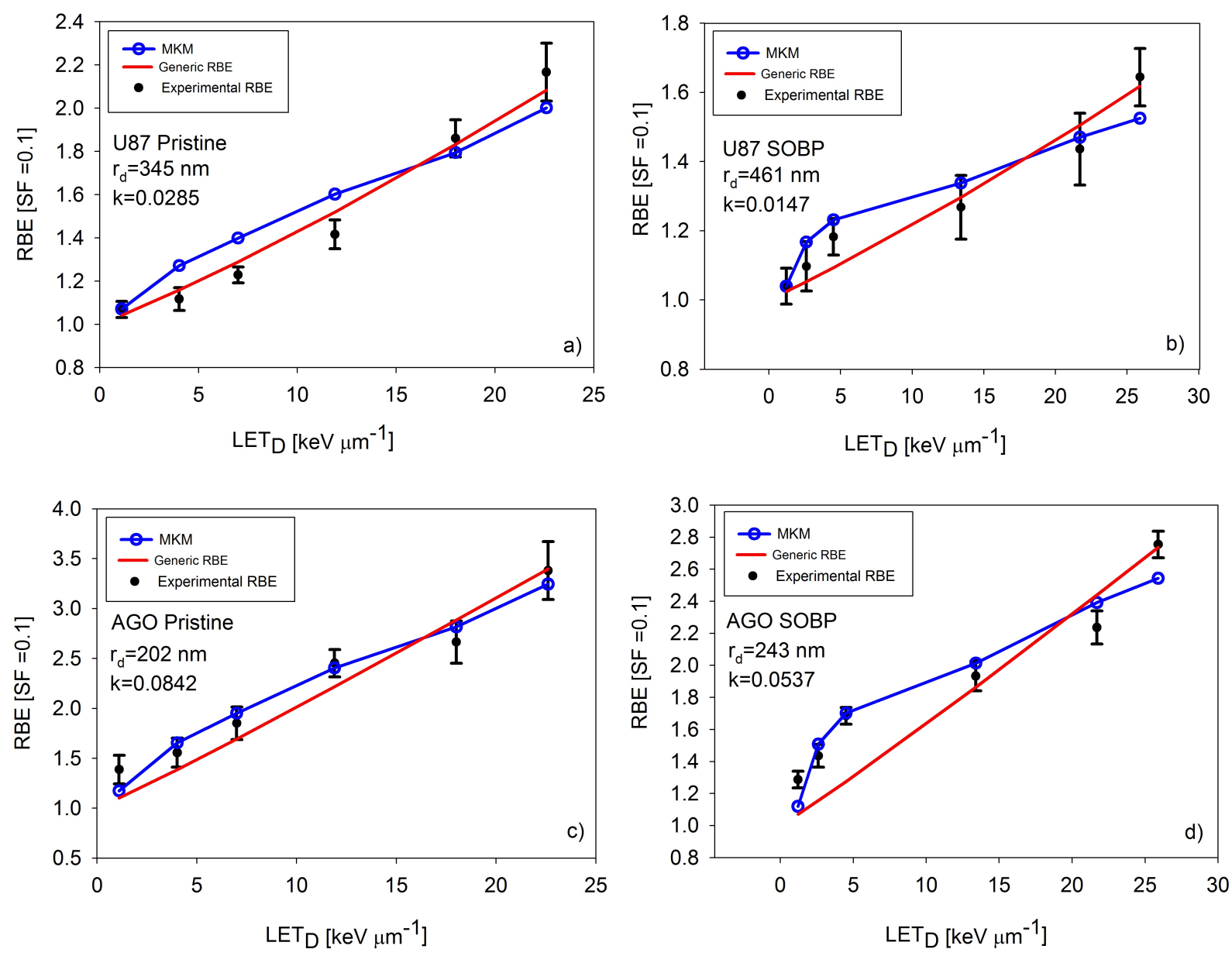


\section{Summary}

Using Monte Carlo simulations, the microdosimetric kinetic model (MKM) was applied to three previously published proton relative biological effectiveness (RBE) studies. The MKM fitting was compared to a generic RBE model as a function of dose averaged linear energy transfer $\left(\mathrm{LET}_{\mathrm{D}}\right)$. Analysis of the fittings reveal no clear advantage to using one model over the other and we conclude that both the MKM and $\mathrm{LET}_{\mathrm{D}}$ based proton RBE models are appropriate. 